\title{
The role of EVI-1 in normal hematopoiesis and myeloid malignancies (Review)
}

\author{
XIAOFEN YUAN $^{1,2^{*}}$, XIDI WANG ${ }^{3 *}$, KEHONG BI $^{4}$ and GUOSHENG JIANG ${ }^{1}$ \\ ${ }^{1}$ Key Laboratory for Rare and Uncommon Diseases of Shandong Province, Institute of Basic Medicine, \\ Shandong Academy of Medical Sciences, Jinan, Shandong; ${ }^{2}$ School of Medicine and Life Sciences, Jinan University, \\ Jinan, Shandong; ${ }^{3}$ Laboratory Department, People's Hospital of Zhangqiu City, Zhangqiu, Shandong; \\ ${ }^{4}$ Department of Hematology, Qianfoshan Hospital of Shandong, Jinan, Shandong, P.R. China
}

Received August 6, 2015; Accepted September 30, 2015

DOI: $10.3892 /$ ijo.2015.3207

\begin{abstract}
Ecotropic virus integration site-1 (EVI-1) gene, locus on chromosome $3(3 q 26.2)$ in the human genome, was first found in the AKXD strain of mice, in a model of retrovirus-induced acute myeloid leukemia (AML) established twenty years ago. Since then, EVI-1 was regarded as one of the most invasive proto-oncogenes in human leukemia. EVI-1 can encode a unique zinc-finger protein of $145 \mathrm{kDa}$ that can bind with DNA, and its overexpression was closely related to human hemopoietic diseases. Furthermore, accumulating research indicates that EVI-1 is involved in the differentiation, apoptosis and proliferation of leukemia cells. The present review focuses on the biochemical properties of EVI-1 which plays a role in myeloid malignancies.
\end{abstract}

\section{Contents}

1. Introduction

2. The structure and downstream product of EVI-1

3. The regulatory effect of EVI-1 on normal hematopoiesis

4. The effect of EVI-1 on erythropoiesis

5. The effect of EVI-1 on granulopoiesis

6. The effect of EVI-1 on megakaryocytopoiesis

7. The relationship between EVI-1 and hematologic neoplasms

Correspondence to: Professor Kehong Bi, Department of Hematology, Qianfoshan Hospital of Shandong, Jinan, Shandong, P.R. China E-mail: bikehong11@163.com

Professor Guosheng Jiang, Key Laboratory for Rare and Uncommon Diseases of Shandong Province, Institute of Basic Medicine, Shandong Academy of Medical Sciences, Jinan, Shandong, P.R. China

E-mail: jianggsh@hotmail.com

${ }^{*}$ Contributed equally

Key words: Ecotropic virus integration site-1, hematopoiesis, myeloid malignancies
8. The molecular mechanisms of EVI-1-mediated leukemogenesis

9. Conclusions

\section{Introduction}

Hematopoiesis is a kind of complex dynamic balance, which is affected by stem cell renewal and lineage-specific differentiation, and that is also regulated by a large number of cytokines, key regulatory transcription factors and epigenetic modifiers $(1,2)$. Leukemias are often induced by rearrangement of chromosomes translocations or inversions. These chromosomal rearrangements can result in gene coding sequence fusions whereby a new protein is created (3-6). For example, the $\mathrm{t}(3 ; 21)(\mathrm{q} 26 ; \mathrm{q} 22)$ translocation results in the RUNX1/MDS1/EVI-1 (AME) transcription factor fusion protein (RUNX1 also termed AML1) (7). The EVI-1 is an oncogenic transcription factor associated with human myeloid malignancy and several solid epithelial cancers (8-11). Aberrant EVI-1 expression occurs in 8-10\% of human adult acute myeloid leukemia (AML) and, strikingly, increases to $27 \%$ of pediatric mixed lineage leukemia (MLL) rearranged leukemias (12). The major question still remains as to its exact role in hemopoietic modulation and leukemogenesis.

\section{The structure and downstream product of EVI-1}

Mucenski and colleagues (13) discovered the murine EVI-1 gene in the DNA products extracted from the leukemic cells of the AKXD mice. The cDNA fragment of human EVI-1 gene is comprised of a 5'-non-coding region (5'-NCR) of $267 \mathrm{bp}$, an open reading frame (ORF) of 3153 bp and a 3 '-non-coding region (3'-NCR) of $167 \mathrm{bp}$. The two ATG codons in the open reading frame are located at the 268 and $290 \mathrm{bp}$, respectively, of which the latter one is located closer to the constant transcription initiation sequence (GCC) GCC(A/G)CCATGG so that the second ATG codon is supposed to be the transcription initiation site (14). The human EVI-1 gene contains 12 domains of which the first one is highly variable and can transcribe 6 different mRNA fragments due to the variablility of its 5 -terminal. It has been shown by Northern blot analysis and 


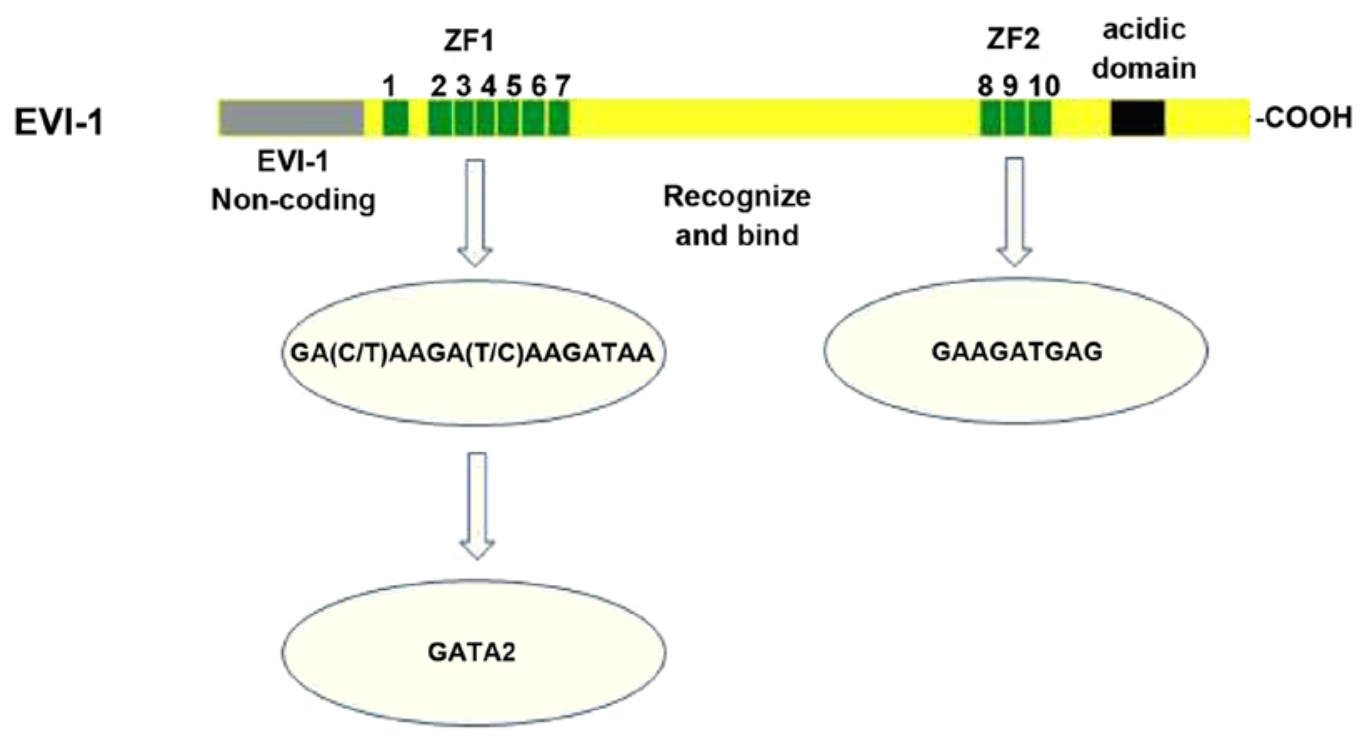

Figure 1. Structure of alternatively spliced forms of EVI-1. EVI-1 is a member of the PR domain family of transcription factors with two sets of the zinc-finger domains.

real-time quantitative RT-PCR that the different types of EVI-1 proteins resulted from the variable 5'-terminal of the EVI-1 gene and are nearly the same in various tissues and are only different in their expression levels in the specific tissues (15). The translation initiation site is located in domain 3 while domain 2 is related to the gene fusion of EVI-1 with MDS1 and AML1. In 1996, Fears et al (16) discovered that there was a MDS1 (myelodysplasia syndrome 1) gene located 170-400 bp upstream of EVI-1 gene. The MDS1 gene was firstly discovered in the myelodysplasia syndromes, and after alternative splicing it can be inserted into the domain 2 of EVI-1, so that the MDS1-EVI-1 fusion transcript is generated and 188 codons are added to the 5'-terminal of the open reading frame in the EVI-1 gene. MDS1 and EVI-1 are mutually antagonistic. Soderholm et al (17) have reported that EVI-1 can inhibit GATA-1-mediated activation and MDS1-EVI-1 is a strong activation sequence containing a GAGA-1 promoter. In addition, the MDS1-EVI-1 fusion gene is also directly inhibited by the normal EVI-1 gene. In the chromosome translocation $\mathrm{t}(3 ; 21)(\mathrm{q} 26 ; \mathrm{q} 22)$ the AML1 gene on chromosome 21 fractures between the runt domain and the transcription activation domain, and the derived fragments will fuse with EVI-1 and MDS1, respectively, thus, the AML1-EVI-1 and AML1-MDS1 fusion genes are formed correspondingly. In the AML1-EVI-1 transcript the exon 5 of AML1 is linked to the second untranslated exon of EVI-1 to encode a fusion protein with the molecular weight of $\sim 1800 \mathrm{k}$ unit and 3 DNA binding domains (DNA-BD). Besides, AML1 canalsofuse with MDS1-EVI-1 to form the AML1-MDS1-EVI-1 fusion gene (1), of which the three members interact with each other with synergistic or antagonistic effects and jointly influence the progression of both human and murine leukemias.

The EVI-1 gene encodes a 145-kDa protein which can be divided into two regions: the region on the $\mathrm{N}$-terminal containing 7 zinc-finger domains and the region on the C-terminal containing 3 zinc-finger domains and a sequence of acidic amino acids, the two of which are separated by a proline-rich region located in the middle (Fig. 1). Delwel et al (18) have reported that in vitro 3 of the zinc-finger domains on the $\mathrm{N}$-terminal can bind with a specific sequence of 15 nucleotides: (C/T)AAGA(T/C)AAGATAA. However, the 3 zinc-finger rings only have an auxiliary function and can just provide a relatively similar sequence of GACAA instead of GATAA for the combination rather than binding with the 15-nucleotide-sequence directly. Funabiki et al (19) have reported that the zinc-finger domains on the $\mathrm{C}$-terminal can recognize and bind a specific sequence of GAAGATGAG. Therefore, EVI-1 is a protein with specific binding sites. EVI-1 protein also has an isotype with a shorter sequence and the molecular weight of $\sim 88 \mathrm{kDa}$ which can be detected both in human being and mice. The $88-\mathrm{kDa}$ isotype lacks zinc-finger domain 6 and zinc-finger 7 which are both essential for DNA binding, so that it has a unique DNA-binding characteristic which is different from that of the $145-\mathrm{kDa}$ EVI-1 protein (20).

\section{The regulatory effect of EVI-1 on normal hematopoiesis}

The ecotropic viral integration site-1 (EVI-1) is an oncogenic transcription factor in murine and human myeloid leukemia. As a proto-oncogene EVI-1 is highly expressed both in human embryo and in the hemopoietic stem cells of the adult bone marrow $(21,22)$. Thus, EVI-1 plays an important role in tissue development and in the proliferation and differentiation of hemopoietic stem cells (HSC) (23). EVI-1 works on the proliferation and differentiation of hemopoietic cells through affecting the expression of GATA-2. When EVI-1 expression on $3 \mathrm{q} 26$ inverted which is misdirected by a distant GATA2 hematopoietic enhancer on 3q21, this kind of enhancer is critical for AML neoplasia (3). It has been reported by Yuasa et al (24) that the hemopoietic stem cells of the EVI-1-deleted mice are deficient in proliferation and repopulation, and also accompanied with low expression level of GATA-2 which is crucial for the proliferation and differentiation of hemopoietic cells. Re-establishing EVI-1 expression 
in the hemopoietic stem cells for these EVI-1-deleted mice can restore the cells' ability of proliferation and differentiation by upregulating GATA-2 expression, and this demonstrates that EVI-1 has the leading role in the proliferation and differentiation of hemopoietic cells. The next in this passage mainly introduces the EVI-1's effects on the various types of hematopoietic progenitor cells. The inhibitor of apoptosis protein survivin regulates hematopoiesis, although its mechanisms of regulation of hematopoietic stem cells (HSCs) remain largely unknown. While investigating conditional survivin deletion in mice, we found that survivin was highly expressed in phenotypically defined HSCs, and survivin deletion in mice resulted in significantly reduced total marrow HSCs and hematopoietic progenitor cells. Transcriptional analysis of survivin $^{-/}$HSCs revealed altered expression of multiple genes not previously linked to survivin activity. In particular, survivin deletion significantly reduced expression of the EVI-1 transcription factor indispensable for HSC function, and the downstream EVI-1 target genes GATA2, Pbx1 and Sall2. The loss of HSCs following survivin deletion and impaired longterm HSC repopulating function could be partially rescued by ectopic EVI-1 expression in survivin ${ }^{-/}$HSCs. These data demonstrate that survivin partially regulates HSC function by modulating the EVI-1 transcription factor and its downstream targets and identify new genetic pathways in HSCs regulated by survivin (22).

\section{The effect of EVI-1 on erythropoiesis}

Since EVI-1 was first identified, many investigators had made a considerable effort to understand the mechanisms how the protein deregulate erythropoiesis. It has been reported by Matsugi et al (25) that the zinc-finger transforming protein of EVI-1 can bind the genomic fragments with the (GATA) $\mathrm{n}$ sequence. The zinc-finger protein GATA-1 is an erythroid transcriptional regulatory factor, and the binding sequences of this kind of factor exist in many cis-acting regulatory elements of the genes expressed in the erythroid cell line, such as the globin gene, the erythropoietin gene and the GATA1 gene. Thus, the GATA-1 protein takes on the central regulator in the specific gene expression and cell differentiation of the erythroid cell line. The binding motif of the zinc-finger domain for binding with GATA-1 is WGATAR (W=A or T, $\mathrm{R}=\mathrm{A}$ or $\mathrm{G}$ ), which is located in the constant binding motif in the N-terminal zinc-finger domains of EVI-1. The effects of EVI-1 on erythropoiesis were confirmed by Kreider et al (26). After the retroviral expressing vectors containing EVI-1 cDNA are successfully transfected into the murine 32DEpol cells in which EVI-1 is not expressed, the transfected EVI-1-expressing cells lose their original response capacity to erythropoietin (EPO) and form far less erythropoietic progenitor colonies than before. Furthermore, the CAT assay has shown that EVI-1 expression can obviously inhibit the CATA-1-dependent transcriptional activation, and the inappropriate expression of EVI-1 probably prohibits erythropoiesis by some subtypes of the GATA-1 target genes. Rather than the DNA sequence, EVI-1 interacts directly with the GATA1 protein. It has been further confirmed that this protein-protein interaction blocks efficient recognition or binding to DNA by GATA1. Point mutations, which destroy the geometry of two zinc-fingers of EVI-1 and terminate the protein-protein interaction, can lead to normal erythroid differentiation of normal murine bone marrow in vitro (27). It has been reported by Louz et al (28) that they established 3 independent transgenic mouse models with the murine Sca-1 promoter, which shows obvious colony forming unit-erythrocyte (CFU-E) reduction in the bone marrow without obvious hemopoietic disorder, and demonstrating the progenitor-related deficiency of normal erythropoiesis. Therefore, we can confirm that EVI-1 can prohibit normal hemopoiesis in vivo which is synergistic with EVI-1-mediated carcinogenesis in return.

\section{The effect of EVI-1 on granulopoiesis}

After EVI-1 is transfected into hematopoietic growth factor (HGF)-dependent murine 32DC13 cells, the granular differentiation induced by granulocyte colony-stimulating factor (G-CSF) is retarded, which demonstrates that EVI-1 can prohibit the maturation of granulocytes. The growth of 32DC13 cells are dependent on interleukin-3 (IL-3) and the cells can differentiate into granulocytes after induction by G-CSF. Morishita et al (29) have reported that with the existence of the hematopoietic growth factors (HGF) the EVI-1-positive 32DC13 cells will lose their original response capacity to G-CSF and undergo further apoptosis, because in the EVI-1-positive 32DC13 cells myeloperoxidase fails to be expressed and the differentiation process is prohibited. Boyd et al (30) reported that the provirus insertion into the EVI-1 gene was observed in the AKZD23 murine leukemia. However, the provirus insertion does not directly cause the occurrence of leukemia, which indicates that there are other synergistic factors. It has been shown by analysis that in every tumor it is the provirus insertion into the SOX4 gene which can encode a high mobility group (HMG) box transcription factor and lead to the overexpression of SOX4. In the 32DC13 cells the overexpression of SOX4 markedly inhibits the cytokine-induced granulocyte maturation. It is believed that the provirus-mediated activation of SOX4 is specifically effective on the provirus-mediated activation of EVI-1, because the reduced process of AKV LTR by SOX4 can upregulate the expression of EVI-1 and lead to the relevant retardation of cell differentiation. EVI-1 is an oncogene inappropriately expressed in the bone marrow (BM) of $\sim 10 \%$ of myelodysplastic syndrome (MDS) patients. This disease is characterized by severe anemia and multilineage myeloid dysplasia that are thought to be a major cause of mortality in MDS patients. We earlier reported on a mouse model that constitutive expression of EVI-1 in the BM led to fatal anemia and myeloid dysplasia, as observed in MDS patients, and we subsequently showed that EVI-1 interaction with GATA1 blocks proper erythropoiesis. Whereas this interaction could provide the basis for the erythroid defects in EVI-1-positive MDS, it does not explain the alteration of myeloid differentiation. In the present review, we have examined the expression of several genes activated during terminal myelopoiesis in BM cells and identified a group that are altered by EVI-1. It was reported that EVI-1 interacts with PU.1 and represses the PU.1-dependent activation of the myeloid promoter. EVI-1 does not seem to inhibit PU.1 binding to DNA but rather to block its association with the coactivator c-Jun. After mapping 
the PU.1-EVI-1 interaction sites, it was shown that an EVI-1 point mutant, unable to bind PU.1, restores the activation of PU.1-regulated genes and allows a normal differentiation of $\mathrm{BM}$ progenitors in vitro (31).

\section{The effect of EVI-1 on megakaryocytopoiesis}

Shimizu et al (32) have reported that in the 3q21q26 syndrome that is characterized by the chromosomal inversion inv(3) (q21;q26), RT-PCR assays can clearly show that EVI-1 is expressed specifically in the $\mathrm{CD} 34^{+}$cells including megakaryocytes and platelets. The immature megakaryoblast leukemia UT-7 cell line has three subtypes including UT-7/GM, UT-7/ EPO and UT-7/TPO, whose growth is dependent on granulocyte macrophage colony-stimulating factor (GM-CSF), erythropoietin (EPO) and thrombopoietin (TPO), respectively. The expression level of the EVI-1 gene is low in the UT-7/ GM and UT-7/EPO cell lines but is high in the UT-7/TPO cell line. After the UT-7/GM cells are stimulated by TPO, the EVI-1 expression is enhanced in these cells accompanied by the increase of multinuclear megakaryocytes and the expression of platelet factor 4 (PF4). The EVI-1 expression in the UT-7/GM cells can change their original morphology into multinuclear megakaryocyte and can lead to cell growth arrest and further cell death in one month, which confirms the effects of EVI-1 expression in megakaryocyte differentiation. Kilbey et al (33) reported the biological effects of EVI-1 expression in the megakaryocytic progenitors and the conditional expression of the EVI-1 gene in the human erythro leukemia (HEL) cells. After the HEL cells are stimulated by $12-O$-Tetradecanoylphorbol-13-acetate (TPA), they will develop toward the megakaryocytic progenitors with four steps: i) growth inhibition; ii) morphological change; iii) endomitosis; and iv) the characteristic change of genetic expression including the reduction of the erythroid-specific glycophorin A and the increase of the megakaryocytic-specific GPIIIa and GPVI. The EVI-1 expression alone has no significant effects on the proliferation and differentiation of the HEL cells, but after the exposure to TPA the EVI-1-expressing HEL cells will undergo obvious growth stagnation along with the alteration of endomitosis and cell morphology. The reduction of glycophoryn A and the increase of the GPIIIa mRNA level can also be detected, while the expression level of the GPVI mRNA can not be increased noticeably. The continuously efficient catalytic activity of CDK2 is specifically related to the endomitosis of megakaryocytes, but in the EVI-1-expressing HEL cells treated with TPA the catalytic activity of CDK2 is remarkably reduced, which is due to the significant reduction in the activity of cyclin A. Although the EVI-1-expressing cells can maintain some molecular characteristics, the decreased catalytic activity of CDK2 can definitely lead to the differentiation deficiency of megakaryocytes via endomitosis retardation and morphological alteration, which confirms the regulatory effects of EVI-1 on megakaryocytopoiesis.

\section{The relationship between EVI-1 and hematologic neoplasms}

Abnormal expression of EVI-1 is common in myelogenous hematopoietic malignancies such as acute myeloid leukemia
(AML), chronic myeloid leukemia (CML) and myelodysplastic syndrome (MDS), and is always caused by transversion or insertion of chromosome 3 which leads to leukemogenesis. Yamazaki et al (3) identified a mechanism whereby a GATA2 distal hematopoietic enhancer (G2DHE or 77-kb enhancer) is brought into close proximity to the EVI-1 gene in inv(3) (q21;q26) inversions, leading to leukemogenesis. They demonstrated that both $3 q$ rearrangements reposition a distal GATA2 enhancer to ectopically activate EVI-1 and simultaneously confer GATA2 functional haploinsufficiency, previously identified as the cause of sporadic familial AML/MDS and MonoMac/Emberger syndromes. Genomic excision of the ectopic enhancer restored EVI-1 silencing and led to growth inhibition and differentiation of AML cells (34). The most common chromosome transversion leading to abnormal EVI-1 expression are chromosome inversion inv(3)(q21;q26) and chromosome transversion $\mathrm{t}(3 ; 3)(\mathrm{q} 21 ; \mathrm{q} 26)$ which account for $7-10 \%$ in the MDS/AML patients. Aberrant EVI-1 expression occurs in $8-10 \%$ of human AML and, strikingly, increased to $27 \%$ of pediatric mixed lineage leukemia (MLL) rearranged leukemias. When inappropriately expressed, EVI-1 is an independent risk factor for a very poor prognosis in hematopoietic cancer (12). In $10 \%$ of the EVI-1-expressing MDS/AML patients the abnormality of chromosome $3 q 26$ is absent, which indicates the existence of synergistic factors in the abnormal expression of EVI-1 and EVI-1-derived hematopoietic malignant transformation. Lahortiqa (ref.?) reported that there are some cases lacking EVI-1 expression in the chromosomal abnormality of inv(3)(q21q26) and $29 \%$ of the AML cases with EVI-1 overexpression lack the variation of chromosome $3 q 26$. In 7 cases of $\operatorname{inv}(3)(q 21 ; q 26)$ or $t(3 ; 3)(q 21 ; q 26)$ or other associated malformations only one case is EVI-1-expressing, which indicates that EVI-1 expression is not adequate to explain all the cases (35). Matsuo et al (36) reported that EVI-1 overexpression is a poor prognostic factor in pediatric patients with mixed lineage leukemia-AF9 rearranged acute myeloid leukemia. Goyama et al (23) developed several leukemia models in which EVI-1 could be deleted, and they found that loss of EVI-1 attenuates proliferative activity in several types of leukemic cells. In particular, EVI-1 deletion led to a large decrease in colony numbers of MLL/ENL-transformed cells, indicating a crucial role for EVI-1 in MLL-leukemia. Because EVI-1 is frequently upregulated in MLL-leukemia, the molecular interaction between EVI-1 and MLL is an important challenge in the future (37).

\section{The molecular mechanisms of EVI-1-mediated leukemo- genesis}

EVI-1 is synergistic with AML1 and MDS1 in the co-induction of hemopoietic stem cell malignant transformation, and the relevant molecular mechanisms mainly include the 7 aspects as follows (Fig. 2):

Inhibition of the AML1-mediated transcriptional activation by EVI-1. AML1-EVI-1 is dominant negative which can exceed and counteract the AML1-mediated transcriptional activation. RUNX1-EVI-1 dominantly suppresses the transactivation capacity of RUNX1 through the PEBP2 sites (38-42). It is reported that the combination of PEBP2 with AML1-EVI-1 


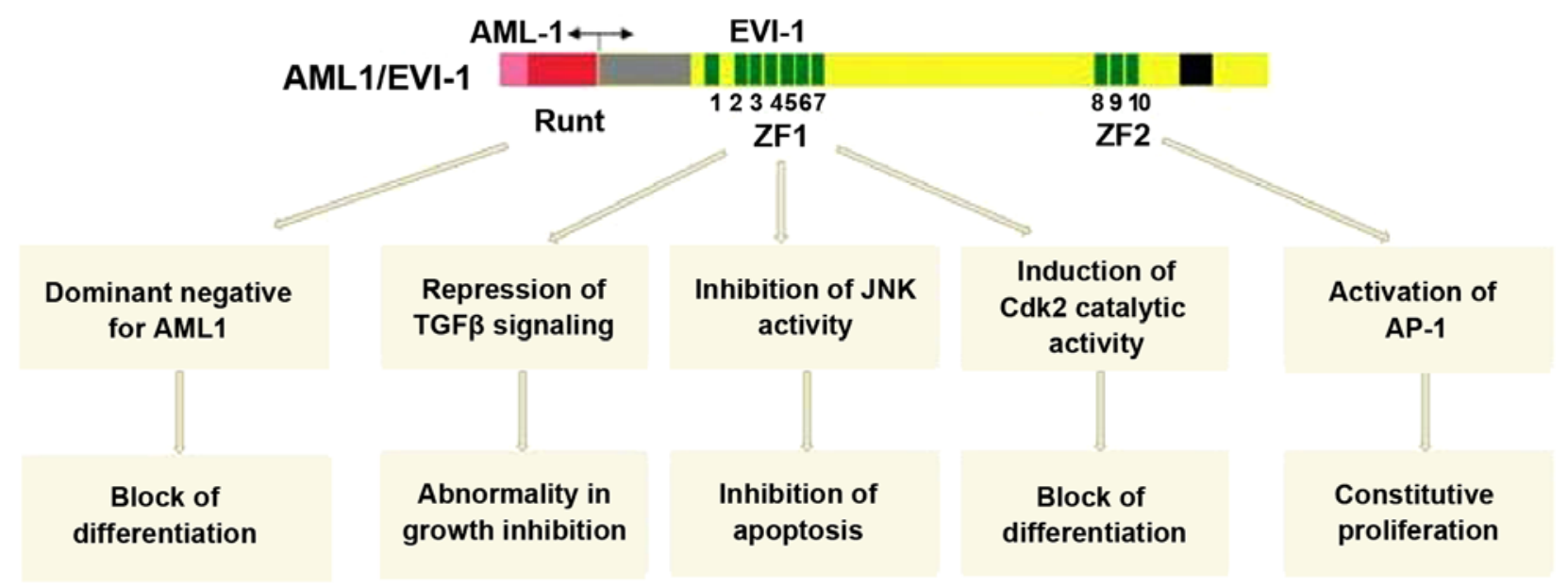

Figure 2. EVI-1 as critical regulator of leukemic cells, there are at least five mechanisms identified in EVI-1 protein than possibly lead into malignant transformation of hematopoietic stem cells.

is tighter than that with AML1, so that AML1-EVI-1 can significantly inhibit the AML1-induced transcriptional activation by competitive combination with specific DNA sequences. To bring about its transcriptional activation function, the AML1 located in the nucleous has to combine its RHD structure with the PEBP2 located in the cytosol. The RHD-positive AML1-EVI-1 can translocate the PEBP2 located in the cytosol to the nucleous, so that AML1-EVI-1 can inhibit the AML1-induced transcriptional activation by means of its higher affinity for PEBP2 (43). It is also reported that AML1-EVI-1 can interact with $\mathrm{COOH}$-terminal-binding protein (CtBP) which is essential for suppressing the AML1induced transcriptional activation. As one of the accessory proteins, CtBP can exert inhibitive effects by combining with several transcriptional factors such as Kruppel-like factor (BKLF), friend of GATA (FOG) and T-cell factor (TCF). Although it is still unclear how CtBP mediates transcriptional inhibition, it is speculated that the interaction between CtBP and histone deacetylase inhibitors (HDACI) is involved in this process. EVI-1 specifically interacts with CtBP via two latent CtBP-binding amino acid sequences located in the AML1-EVI-1 including the PFDLT sequence on the $\mathrm{N}$-terminal and the PLDLS sequence on the C-terminal, one of which interacts with CtBP directly. AML1-EVI-1 can interfere with AML1-induced transcription, but the AML1-EVI-1 with changed PLDLS fails to do so, which confirms that the function of CtBP as a co-repressor is essential in the AML1EVI-1-mediated inhibition of AML1-induced transcription. As a histone deacetylase inhibitor (HDACI), trichostatin A can diminish the dominant negative effect of AML1-EVI-1, therefore, AML1-EVI-1 probably resorts to the CtBP-mediated recruitment of HDACI in order to prohibit the AML1-induced transcription (44-46).

EVI-1 counteracts the TGF- $\beta 1$ signaling pathway. EVI-1 can interrupt the TGF- $\beta 1 /$ SMAD signaling pathway and counteracts the growth inhibition caused by TGF- $\beta 1$ by inhibiting the TGF- $\beta 1 /$ SMAD3-mediated transcription. EVI-1 albumen which depends on the first zinc finger domain of itself can integrate with the phosphorylation of Smad3 of MH2 structural domain and collect other transcription repressors (including CtBP, histone deacetylase) accordingly, it impedes the function of accelerating target gene transcription of phosphorylation Smad3, that according to the MH2 structural domain cooperated with other activating transcription factors. As a result, during the period of cell loses the normal inhibitory control leads to the cell maintaining excessive growth and proliferation making an obstacle for terminal differentiation and apoptosis of cells. Finally, this process results in the generation of cancer cells (47-49). One of the histone deacetylase inhibitors (HDACI), trichostatin A, can diminish the EVI-1-mediated inhibition of the TGF- $\beta$-mediated signal transduction, which indicates that histone deacetylase (HDAC) is essential in this process $(50,51)$. However, Liu et al (52) discovered that the continuous EVI-1 expression in the non-transformed intestinal epithelial cells can inhibit some SMAD3-dependent target genes of TGF- $\beta 1$ such as PAI1, but the sensitivity of the intercellular supportive substance such as integrin1 and paxillin in response to TGF- $\beta 1$ induction as well as the transformation of the epithelial cells into the mesenchyme cells can not be inhibited by EVI-1. Moreover, EVI-1 could not inhibit the downregulation of cyclin D1 expression and cell growth inhibition induced by TGF- $\beta 1$. But EVI-1 can interact with P13K and its downstream effector AKT to inhibit the TGF- $\beta$ induced apoptosis. The ability of EVI-1 to inhibit apoptosis is not confined to the TGF- $\beta$-induced cell death, because EVI-1 also has a protective effect on the intestinal epithelial cells and can inhibit the paclitaxel-induced apoptosis as well. EVI-1 is overexpressed in some human clonal tumor cells. The siRNA-mediated knockout of the EVI-1 gene in the HT-29 human clonal tumor cells can inhibit AKT phosphorylation and enhance the sensitivity of these cells to paclitaxel-induced apoptosis, which confirms that in the intestinal epithelial cells and the clonal tumor cells EVI-1 acts as a survival gene that can activate P13K/AKT and counteract the apoptosis caused by physiological or therapeutic factors.

EVI-1 modulates also other transcription factors. EVI-1 acts as an inhibitor of JNK (43), which can inhibit the phosphorylase activity of C-jun expression, while the activity of JNK is 
dependent on the phosphorylation of two specific amino acid residues (Thr183 and Tyr185) by MMK4 or MMK7. EVI-1 can interact with JNK, yet, EVI-1 does not affect the phosphorylation level of JNK. EVI-1 expression can interrupt the binding between $\mathrm{C}$-jun and JNK so that the phosphorylation of C-jun can be inhibited. EVI-1 can also resort to affect the JNK-mediated signaling pathway to inhibit cell apoptosis. The human embryonic kidney 293 cells will undergo apoptosis after induction by ultraviolet radiation, but the counterpart cells with EVI-1 overexpression are markedly resistant to apoptosis. The human endometrial carcinoma HEC1B cells are resistant to apoptosis, which is probably due to the innate EVI-1 expression (53).

As to the upregulation of AP-1 activity by EVI-1, Hirai et al (48) found that EVI-1 can upregulate reporter constructs bearing the AP-1-responsive promoter region. AP-1 activity (Fos/Jun heterodimer) is activated by a variety of growth signals, including phorbol esters [12- $O$-tetradecanoylphorbol 13-acetate (TPA)]. Tanaka et al (54) showed that EVI-1 could markedly upregulate porter constructs with TPA-responsive elements (TRE). Similarly, this group also showed that RUNX1-MDS1-EVI-1 (RME), the protein product of the leukemia-associated $\mathrm{t}(3 ; 21)$, can also transactivate a TRE reporter. Through structure-activity-relationship (SAR) studies, it was shown that activation of AP-1 requires the second set of zinc fingers (zinc fingers 8-10; ZF2), and appears to occur via transcriptional upregulation of Fos, via an element within the promoter that does not appear to bind EVI-1. The exact mechanism of Fos upregulation is not clear; nor is it clear whether AP-1 uregulation is required for leukemogenesis. Noting that transcriptional activation of Fos is lost upon deletion of $\mathrm{ZF} 2$, it is important to consider that neither transformation of bone marrow progenitors by RME nor transformation of Rat1 fibroblasts require DNA binding via $\mathrm{ZF} 2$ (55). It is possible that a non-DNA binding function of $\mathrm{ZF} 2$ is required for both Fos activation and transformation.

Downregulation of Cdk2 kinase activity. The EVI-1 transcriptional repressor domain and the ZF1 DNA binding domain are required for both cell transformation and induction of Cdk2 catalytic activity. EVI-1 expression represses the transcription of target genes, which may include p27 that deregulate the normal control of the G1 phase of the cell cycle, providing a cellular proliferative advantage that contributes to transformation in vitro and leukemogenesis in vivo (56). Karakaya et al (57) suggest that EVI-1 overexpression leads to activation of $\mathrm{p} 53$ and subsequent upregulation of $\mathrm{p} 21$, activation of the tetraploidy checkpoint appears to be underlying cause of p53 activation, since both p53 and p21 are most abundant in enlarged nuclei. EVI-1 overexpression is mechanistically linked to the p53 pathway or cytokinesis failure as the underlying cause of activation of the tetraploidy checkpoint. Thus, Cdk2 inhibition and consequential G0/1 arrest were related to EVI-1 expression. Kilbey et al (33) conclude that CDK2 kinase activity was substantially reduced in TPA-induced HEL cells in the presence of EVI-1 and the profile of kinase activity directly correlated with the abundance of cyclin A.

Epigenetic aberrations of EVI-1. There is a growing body of evidence for epigenetic aberrations associated with
EVI-1-induced leukemia (57-59). In patients with AML and MDS, increased expression of EVI-1 was found to be associated with adverse prognosis. Although increased expression of the EVI-1 gene is mainly caused by chromosomal rearrangements involving chromosome band 3q26, where EVI-1 is located, it can also be identified in cases without these rearrangements. Overexpression of EVI-1 is associated with $3 q 26$ chromosomal rearrangements and confers extremely poor prognosis in AML. EVI-1 amplification on cytogenetically cryptic dmin causes increased expression of EVI-1 and is a new mechanism that causes increased EVI-1 expression without a 3q26 rearrangement (61). A study (58) on 476 AML patients reported that those with no basal expression of the EVI-1 gene have a better prognosis than patients with EVI-1 overexpression. Consistent with the fact that DNA methylation is generally associated with repression of gene transcription, EVI-1 overexpression is associated with the absence of DNA methylation in the promoter region of EVI-1. The EVI-1 locus is also enriched with marks of active gene transcription, specifically histones $\mathrm{H} 3$ and $\mathrm{H} 4$ acetylation, and histone $\mathrm{H} 3$ lysine 4 (H3K4) trimethylation. Conversely, cells without EVI-1 expression are associated with DNA hypermethylation and marks of gene repression such as trimethylation of histone $\mathrm{H} 3$ lysine 27 (H3K27) at the EVI-1 promoter (7). Lugthart et al (62) showed that AML samples with activated EVI-1 presented with a deregulated hypermethylation signature, possibly through physical interactions between EVI-1 and the DNA methyltransferases DNMT3A and DNMT3B. Yoshimi and Kurokawa (63) found that EVI-1 activated the AKT/mTOR signaling pathway through transcriptional repression of PTEN. The activation of this signaling is essential for EVI-1-mediated leukemogenesis. All of the above jointly accelerate the malignant transformation of the hemopoietic stem cells.

The minimal promoter region of EVI-1 demonstrates that RUNX1 and ELK1, its promoter region of EVI-1 of $318 \mathrm{bp}$, two proteins with essential functions in hematopoiesis, regulate EVI-1 in AML. One of the mechanisms by which RUNX1 regulates the transcription of EVI-1 is by acetylation of the histone $\mathrm{H} 3$ on its promoter region. The transcription factors RUNX1 and ELK1 are involved in the transcriptional regulation of this gene, and that RUNX1 regulates EVI-1 during megakaryocyte differentiation (64). H3 and H4 acetylation, and trimethylation of histone $\mathrm{H} 3$ lysine 4 and histone $\mathrm{H} 3$ lysine 27 might play a role in the transcriptional regulation of EVI-1 in acute myeloid leukemias (58). This result opens new directions to further understand the mechanisms of EVI-1 overexpressing leukemias (59). The EVI-1 gene, located in chromosomal band $3 \mathrm{q} 26$, is a transcription factor that has stem cell-specific expression pattern and is essential for the regulation of self-renewal of hematopoietic stem cells. It is now recognized as one of the dominant oncogenes associated with myeloid leukemia. EVI-1 overexpression is associated with minimal to no response to chemotherapy and poor clinical outcome. Several chromosomal rearrangements involving band $3 q 26$ are known to induce EVI-1 overexpression. They are mainly found in acute myeloid leukemia and blastic phase of Philadelphia chromosome-positive chronic myeloid leukemia, more rarely in myelodysplastic syndromes. They include inv(3) (q21q26), t(3;3)(q21;q26), t(3;21)(q26;q22), t(3;12)(q26;p13) and 
$\mathrm{t}(2 ; 3)(\mathrm{p} 15-23 ; \mathrm{q} 26)$. However, many other chromosomal rearrangements involving 3q26/EVI-1 have been identified. The precise molecular event has not been elucidated in the majority of these chromosomal abnormalities and most gene partners remain unknown.

Dysregulated T-cell leukemia/lymphoma-1A (TCL1A), a modulator in B-cell receptor (BCR) signaling, is causally implicated in chronic lymphocytic leukemia (CLL). However, the mechanisms of the perturbed TCL1A regulation are largely unknown. To characterize TCL1A-upstream networks, we functionally screened for TCL1A-repressive micro-RNAs (miRs) and their transcriptional regulators. They identified the novel miR-484 to target TCL1A's 3'-UTR and to be downregulated in CLL (ref.?). In chromatin immunoprecipitations and reporter assays, the oncogenic transcription factor of myeloid cells, EVI-1, bound and activated the miR-484 promoter. Most common in CLL was a pan-EVI-1 transcript variant. EVI-1 protein expression revealed distinct normal-tissue and leukemia-associated patterns of EVI-1/TCL1A co-regulation. EVI-1 levels were particularly low in TCL1A-high CLL or such cellular subsets. Global gene expression profiles from a 337-patient set linked EVI-1 networks to BCR signaling and cell survival via TCL1A, BTK and other molecules of relevance in CLL. Enforced EVI-1, similar to miR-484, repressed TCL1A. Furthermore, it reduced phospho-kinase levels, impaired cell survival, mitigated BCR-induced Ca-flux and diminished the in vitro ibrutinib response. Moreover, TCL1A and EVI-1 showed a strongly interactive hazard prediction in prospectively treated patients. Overall, the regressive EVI-1 is a novel regulatory signature in CLL. Through enhanced TCL1A and other EVI-1-targeted hallmarks of CLL, this contributes to an aggressive cellular and clinical phenotype $(65,66)$.

The development of novel technologies, such as massively parallel DNA sequencing, has led to the identification of several novel recurrent gene mutations, such as DNA methyltransferase (Dnmt)3a, ten-eleven-translocation oncogene family member 2 (TET2), isocitrate dehydrogenase (IDH)1/2, additional sex comb-like 1 (ASXL1), enhancer of zeste homolog 2 (EZH2) and ubiquitously transcribed tetratricopeptide repeat X chromosome (UTX) mutations in AML and other myeloid malignancies. These findings strongly suggest a link between recurrent genetic alterations and aberrant epigenetic regulations, resulting from an abnormal DNA methylation and histone modification status. Moreover, epigenetic aberrations resulting from transcription factor aberrations, such as MLL rearrangement, EVI-1 overexpression, chromosomal translocations and the downregulation of PU.1 are also described (67).

EVI-1 and MEL1 are homolog genes whose transcriptional activations by chromosomal translocations are known in small subsets of leukemia. From gene expression profiling data of 130 Japanese pediatric AML patients, we found that EVI-1 and MEL1 were overexpressed in $30 \%$ of patients without obvious translocations of these gene loci, and that their high expression was significantly associated with inferior survival. High EVI-1 expression was detected mainly in myelomonocyticlineage (designated as e-M4/M5 subtype) leukemia with MLL rearrangements and in megakaryocytic-lineage (designated as e-M7 subtype) leukemia, and its prognostic association was observed in the e-M4/M5 subtype but not in the e-M7 subtype.
On the other hand, high MEL1 expression was detected in myelocytic-lineage (designated as e-M0/M1/M2 subtype) and e-M4/M5 subtype leukemia without MLL rearrangements, and its prognostic association was independent from the subtypes. The combined estimation of EVI-1 and MEL1 expression will be an effective method to predict the prognosis of pediatric AML (68). The genetic and transcriptional signature of EVI-1-rearranged (EVI-1-r) AMLs remains poorly defined. EVI-1-r AMLs are also characterized by a unique transcriptional signature with high expression levels of MECOM, PREX2, VIP, MYCT1 and PAWR. EVI-1-r AMLs could be molecularly defined by specific transcriptomic anomalies and a hitherto unseen mutational pattern. Larger patient cohorts will better determine the frequency of these events (69).

\section{Conclusions}

At present, it has become evident that EVI-1 is a crucial gene for HSC regulation as well as an oncogene in development of leukemia. The abnormal expression of it could predict poor prognosis in hematological malignancies. Since EVI-1 overexpression was closely related to human hemopoietic diseases, which was involved in the differentiation, apoptosis and proliferation of leukemia cells, certain drugs or antisense oligonucleotides are expected to be good methods to treat EVI-1 induced malignancies, for example, HDAC inhibitors might be useful in the treatment of EVI-1 induced leukemias.

\section{Acknowledgements}

The present review was supported by grants from the National Natural Science Foundation of China (nos. 81300426, 30771103, 815734467 and 81172792), the Project for Shandong Medical and Health science and Technology Plan Project (2013WS0365), Natural Science Foundation of Shandong (ZR2015HM014), and the 'Twelfth Five-Year' National Science and Technology Support Program (2013BAI07B02).

\section{References}

1. Nucifora G, Laricchia-Robbio L and Senyuk V: EVI1 and hematopoietic disorders: History and perspectives. Gene 368: 1-11, 2006.

2. Haladyna JN, Yamauchi T, Neff T and Bernt KM: Epigenetic modifiers in normal and malignant hematopoiesis. Epigenomics 7: 301-320, 2015.

3. Yamazaki H, Suzuki M, Otsuki A, Shimizu R, Bresnick EH, Engel JD and Yamamoto M: A remote GATA2 hematopoietic enhancer drives leukemogenesis in $\operatorname{inv}(3)(\mathrm{q} 21 ; \mathrm{q} 26)$ by activating EVI-1 expression. Cancer Cell 25: 415-427, 2014.

4. Ney Garcia DR, Liehr T, Emerenciano M, Meyer C, Marschalek R, Pombo-de-Oliveira MS, Ribeiro RC, Poirot Land MG and Macedo Silva ML: Molecular studies reveal a MLL-MLLT3 gene fusion displaced in a case of childhood acute lymphoblastic leukemia with complex karyotype. Cancer Genet 208: 143-147, 2015.

5. De Braekeleer M, Le Bris MJ, De Braekeleer E, Basinko A, Morel F and Douet-Guilbert N: 3q26/EVI-1 rearrangements in myeloid hemopathies: A cytogenetic review. Future Oncol 11: 1675-1686, 2015.

6. Su G, Lian X, Tan D, Tao H, Liu H, Chen S, Yin H, Wu D and Yin B: Aberrant expression of ecotropic viral integration site-1 in acute myeloid leukemia and acute lymphoblastic leukemia. Leuk Lymphoma 56: 472-479, 2015.

7. Glass C, Wilson M, Gonzalez R, Zhang Y and Perkins AS: The role of EVI1 in myeloid malignancies. Blood Cells Mol Dis 53: 67-76, 2014. 
8. Koos B, Bender S, Witt H, Mertsch S, Felsberg J, Beschorner R, Korshunov A, Riesmeier B, Pfister S, Paulus W, et al: The transcription factor evi-1 is overexpressed, promotes proliferation, and is prognostically unfavorable in infratentorial ependymomas. Clin Cancer Res 17: 3631-3637, 2011.

9. Jazaeri AA, Ferriss JS, Bryant JL, Dalton MS and Dutta A: Evaluation of EVI-1 and EVI-1s (Delta324) as potential therapeutic targets in ovarian cancer. Gynecol Oncol 118: 189-195, 2010.

10. Balgobind BV, Lugthart S, Hollink IH, Arentsen-Peters ST, van Wering ER, de Graaf SS, Reinhardt D, Creutzig U, Kaspers GJ, de Bont ES, et al: EVI-1 overexpression in distinct subtypes of pediatric acute myeloid leukemia. Leukemia 24 942-949, 2010

11. Yasui K, Konishi C, Gen Y, Endo M, Dohi O, Tomie A, Kitaichi T, Yamada N, Iwai N, Nishikawa T, et al: EVI-1, a target gene for amplification at 3q26, antagonizes transforming growth factor- $\beta$ mediated growth inhibition in hepatocellular carcinoma. Cancer Sci 106: 929-937, 2015.

12. Bindels EMJ, Havermans M, Lugthart S, Erpelinck C, Wocjtowicz E, Krivtsov AV, Rombouts E, Armstrong SA, Taskesen E, Haanstra JR, et al: EVI-1 is critical for the pathogenesis of a subset of MLL-AF9-rearranged AMLs. Blood 119: 5838-5849, 2012.

13. Mucenski ML, Taylor BA, Ihle JN, Hartley JW, Morse HC III, Jenkins NA and Copeland NG: Identification of a common ecotropic viral integration site, EVI-1, in the DNA of AKXD murine myeloid tumors. Mol Cell Biol 8: 301-308, 1988.

14. Xu K, Wang L and Hao Y: Advances in the study of EVI-1 and mds1 genes. Zhonghua Xue Ye Xue Za Zhi 20: 331-333, 1999 (In Chinese).

15. Aytekin M, Vinatzer U, Musteanu M, Raynaud S and Wieser R: Regulation of the expression of the oncogene EVI-1 through the use of alternative mRNA 5'-ends. Gene 356: 160-168, 2005.

16. Fears S, Mathieu C, Zeleznik-Le N, Huang S, Rowley JD and Nucifora G: Intergenic splicing of MDS1 and EVI-1 occurs in normal tissues as well as in myeloid leukemia and produces a new member of the PR domain family. Proc Natl Acad Sci USA 93: 1642-1647, 1996.

17. Soderholm J, Kobayashi H, Mathieu C, Rowley JD and Nucifora G: The leukemia-associated gene MDS1/EVI-1 is a new type of GATA-binding transactivator. Leukemia 11: 352-358, 1997.

18. Delwel R, Funabiki T, Kreider BL, Morishita K and Ihle JN: Four of the seven zinc fingers of the EVI-1 myeloid-transforming gene are required for sequence-specific binding to $\mathrm{GA}(\mathrm{C} / \mathrm{T})$ AAGA(T/C)AAGATAA. Mol Cell Biol 13: 4291-4300, 1993.

19. Funabiki T, Kreider BL and Ihle JN: The carboxyl domain of zinc fingers of the EVI-1 myeloid transforming gene binds a consensus sequence of GAAGATGAG. Oncogene 9: 1575-1581, 1994.

20. Lopingco MC and Perkins AS: Molecular analysis of EVI-1, a zinc finger oncogene involved in myeloid leukemia. Curr Top Microbiol Immunol 211: 211-222, 1996.

21. Saito Y and Morishita K: Maintenance of leukemic and normal hematopoietic stem cells in bone marrow niches by EVI-1regulated GPR56. Rinsho Ketsueki 56: 375-383, 2015 (In Japanese).

22. Fukuda S, Hoggatt J, Singh P, Abe M, Speth JM,Hu P, Conway EM, Nucifora G, Yamaguchi S and Pelus LM: Survivin modulates genes with divergent molecular functions and regulates proliferation of hematopoietic stem cells through EVI-1. Leukemia 29: 433-440, 2015.

23. Goyama S, Yamamoto G, Shimabe M, Sato T, Ichikawa M, Ogawa S, Chiba S and Kurokawa M: EVI-1 is a critical regulator for hematopoietic stem cells and transformed leukemic cells. Cell Stem Cell 3: 207-220, 2008

24. Yuasa H, Oike Y, Iwama A, Nishikata I, Sugiyama D, Perkins A, Mucenski ML, Suda T and Morishita K: Oncogenic transcription factor EVI-1 regulates hematopoietic stem cell proliferation through GATA-2 expression. EMBO J 24: 1976-1987, 2005.

25. Matsugi T, Kreider BL, Delwel R, Cleveland JL, Askew DS and Ihle JN: The EVI-1 zinc finger myeloid transforming protein binds to genomic fragments containing (GATA)n sequences. Oncogene 11: 191-198, 1995.

26. Kreider BL, Orkin SH and Ihle JN: Loss of erythropoietin responsiveness in erythroid progenitors due to expression of the EVI-1 myeloid-transforming gene. Proc Natl Acad Sci USA 90: 6454-6458, 1993.
27. Laricchia-Robbio L, Fazzina R, Li D, Rinaldi CR, Sinha KK, Chakraborty S and Nucifora G: Point mutations in two EVI-1 Zn fingers abolish EVI-1-GATA1 interaction and allow erythroid differentiation of murine bone marrow cells. Mol Cell Biol 26: 7658-7666, 2006

28. Louz D, van den Broek M, Verbakel S, Vankan Y, van Lom K, Joosten M, Meijer D, Löwenberg B and Delwel R: Erythroid defects and increased retrovirally-induced tumor formation in EVI-1 transgenic mice. Leukemia 14: 1876-1884, 2000.

29. Morishita K, Parganas E, Matsugi T and Ihle JN: Expression of the EVI-1 zinc finger gene in 32Dc13 myeloid cells blocks granulocytic differentiation in response to granulocyte colonystimulating factor. Mol Cell Biol 12: 183-189, 1992.

30. Boyd KE, Xiao YY, Fan K, Poholek A, Copeland NG, Jenkins NA and Perkins AS: Sox4 cooperates with EVI-1 in AKXD-23 myeloid tumors via transactivation of proviral LTR. Blood 107: 733-741, 2006.

31. Laricchia-Robbio L, Premanand K, Rinaldi CR and Nucifora G: EVI-1 Impairs myelopoiesis by deregulation of PU.1 function. Cancer Res 69: 1633-1642, 2009.

32. Shimizu S, Nagasawa T, Katoh O, Komatsu N, Yokota J and Morishita K: EVI-1 is expressed in megakaryocyte cell lineage and enforced expression of EVI-1 in UT-7/GM cells induces megakaryocyte differentiation. Biochem Biophys Res Commun 292: 609-616, 2002.

33. Kilbey A, Alzuherri H, McColl J, Calés C, Frampton J and Bartholomew C: The EVI-1 proto-oncoprotein blocks endomitosis in megakaryocytes by inhibiting sustained cyclin-dependent kinase 2 catalytic activity. Br J Haematol 130: 902-911, 2005.

34. Gröschel S, Sanders MA, Hoogenboezem R, de Wit E, Bouwman BA, Erpelinck C, van der Velden VH, Havermans M, Avellino R, van Lom K, et al: A single oncogenic enhancer rearrangement causes concomitant EVI-1 and GATA2 deregulation in leukemia. Cell 157: 369-381, 2014.

35. Lahortiga I, Vázquez I, Agirre X, Larrayoz MJ, Vizmanos JL, Gozzetti A, Calasanz MJ and Odero MD: Molecular heterogeneity in AML/MDS patients with 3q21q26 rearrangements. Genes Chromosomes Cancer 40: 179-189.

36. Matsuo H, Kajihara M, Tomizawa D, Watanabe T, Saito AM, Fujimoto J, Horibe K, Kodama K, Tokumasu M, Itoh $\mathrm{H}$, et al: EVI-1 overexpression is a poor prognostic factor in pediatric patients with mixed lineage leukemia-AF9 rearranged acute myeloid leukemia. Haematologica 99: e225-e227, 2014.

37. Goyama S and Kurokawa M: EVI-1 as a critical regulator of leukemic cells. Int J Hematol 91: 753-757, 2010.

38. Ito Y: Oncogenic potential of the RUNX gene family: 'overview'. Oncogene 23: 4198-4208, 2004.

39. van Wijnen AJ, Stein GS, Gergen JP, Groner Y, Hiebert SW, Ito Y, Liu P, Neil JC, Ohki M and Speck N: Nomenclature for Runt-related (RUNX) proteins. Oncogene 23: 4209-4210, 2004.

40. Levanon D and Groner Y: Structure and regulated expression of mammalian RUNX genes. Oncogene 23: 4211-4219, 2004.

41. Durst KL and Hiebert SW: Role of RUNX family members in transcriptional repression and gene silencing. Oncogene 23: 4220-4224, 2004.

42. Cameron ER and Neil JC: The Runx genes: Lineage-specific oncogenes and tumor suppressors. Oncogene 23: 4308-4314, 2004.

43. Mitani K: Molecular mechanisms of leukemogenesis by AML1/ EVI-1. Oncogene 23: 4263-4269, 2004.

44. Palmer S, Brouillet JP, Kilbey A, Fulton R, Walker M, Crossley M and Bartholomew C: EVI-1 transforming and repressor activities are mediated by CtBP co-repressor proteins. J Biol Chem 276: 25834-25840, 2001.

45. Senyuk V, Chakraborty S, Mikhail FM, Zhao R, Chi Y and Nucifora G: The leukemia-associated transcription repressor AML1/MDS1/EVI-1 requires CtBP to induce abnormal growth and differentiation of murine hematopoietic cells. Oncogene 21: 3232-3240, 2002.

46. Chinnadurai G: $\mathrm{CtBP}$, an unconventional transcriptional corepressor in development and oncogenesis. Mol Cell 9: 213-224, 2002.

47. Kahata K, Asaka M and Miyazono K: TGF-beta signaling and carcinogenesis. Nihon Rinsho 63 (Suppl 4): 549-554, 2005 (In Japanese).

48. Hirai H, Izutsu K, Kurokawa M and Mitani K: Oncogenic mechanisms of EVI-1 protein. Cancer Chemother Pharmacol 48 (Suppl 1): S35-S40, 2001.

49. Alliston T, Ko TC, Cao Y, Liang YY, Feng XH, Chang C and Derynck R: Repression of bone morphogenetic protein and activin-inducible transcription by EVI-1. J Biol Chem 280: 24227-24237, 2005. 
50. Izutsu K, Kurokawa M, Imai Y, Maki K, Mitani K and Hirai H: The corepressor CtBP interacts with EVI-1 to repress transforming growth factor $\beta$ signaling. Blood 97: 2815-2822, 2001.

51. Vinatzer U, Taplick J, Seiser C, Fonatsch C and Wieser R: The leukaemia-associated transcription factors EVI-1 and MDS1/ EVI-1 repress transcription and interact with histone deacetylase. Br J Haematol 114: 566-573, 2001.

52. Liu Y, Chen L, Ko TC, Fields AP and Thompson EA: EVI-1 is a survival factor which conveys resistance to both TGFbetaand taxol-mediated cell death via PI3K/AKT. Oncogene 25: 3565-3575, 2006.

53. Kurokawa M, Mitani K, Yamagata T, Takahashi T, Izutsu K, Ogawa S, Moriguchi T, Nishida E, Yazaki Y and Hirai H: The EVI-1 oncoprotein inhibits c-Jun N-terminal kinase and prevents stress-induced cell death. EMBO J 19: 2958-2968, 2000.

54. Tanaka T, Nishida J, Mitani K, Ogawa S, Yazaki Y and Hirai H: EVI-1 raises AP-1 activity and stimulates c-fos promoter transactivation with dependence on the second zinc finger domain. J Biol Chem 269: 24020-24026, 1994.

55. Zhang Y, Sicot G, Cui X, Vogel M, Wuertzer CA, Lezon-Geyda K, Wheeler J, Harki DA, Muzikar KA, Stolper DA, et al: Targeting a DNA binding motif of the EVI-1 protein by a pyrrole-imidazole polyamide. Biochemistry 50: 10431-10441, 2011.

56. Kilbey A, Stephens V and Bartholomew C: Loss of cell cycle control by deregulation of cyclin-dependent kinase 2 kinase activity in EVI-1 transformed fibroblasts. Cell Growth Differ 10 601-610, 1999.

57. Karakaya K, Herbst F, Ball C, Glimm H, Krämer A and Löffler H: Overexpression of EVI-1 interferes with cytokinesis and leads to accumulation of cells with supernumerary centrosomes in $\mathrm{G} 0 / 1$ phase. Cell Cycle 11: 3492-3503, 2012.

58. Pradhan AK, Mohapatra AD, Nayak KB and Chakraborty S: Acetylation of the proto-oncogene EVI-1 abrogates Bcl-xL promoter binding and induces apoptosis. PLoS One 6: e25370, 2011.

59. Vázquez I, Maicas M, Cervera J, Agirre X, Marin-Béjar O, Marcotegui N, Vicente C, Lahortiga I, Gomez-Benito M, Carranza C, et al: Down-regulation of EVI-1 is associated with epigenetic alterations and good prognosis in patients with acute myeloid leukemia. Haematologica 96: 1448-1456, 2011.
60. White DJ, Unwin RD, Bindels E, Pierce A, Teng HY, Muter J, Greystoke B, Somerville TD, Griffiths J, Lovell S, et al: Phosphorylation of the leukemic oncoprotein EVI-1 on serine 196 modulates DNA binding, transcriptional repression and transforming ability. PLoS One 8: e66510, 2013.

61. Volkert S, Schnittger S, Zenger M, Kern W, Haferlach T and Haferlach C: Amplification of EVI-1 on cytogenetically cryptic double minutes as new mechanism for increased expression of EVI-1. Cancer Genet 207: 103-108, 2014.

62. Lugthart S, Figueroa ME, Bindels E, Skrabanek L, Valk PJ, Li Y, Meyer S, Erpelinck-Verschueren C, Greally J, Löwenberg B, et al: Aberrant DNA hypermethylation signature in acute myeloid leukemia directed by EVI-1. Blood 117: 234-241, 2011.

63. Yoshimi A and Kurokawa M: EVI-1 forms a bridge between the epigenetic machinery and signaling pathways. Oncotarget 2: 575-586, 2011.

64. Maicas M, Vázquez I, Vicente C, García-Sánchez MA, Marcotegui N, Urquiza L, Calasanz MJ and Odero MD: Functional characterization of the promoter region of the human EVI-1 gene in acute myeloid leukemia: RUNX1 and ELK1 directly regulate its transcription. Oncogene 32: 2069-2078, 2013.

65. Vasyutina E, Boucas JM, Bloehdorn J, Aszyk C, Crispatzu G, Stiefelhagen M, Breuer A, Mayer P, Lengerke C, Döhner H, et al: The regulatory interaction of EVI-1 with the TCL1A oncogene impacts cell survival and clinical outcome in CLL. Leukemia 10: $1038,2015$.

66. Matsuo H, Goyama S, Kamikubo Y and Adachi S: The subtypespecific features of EVI-1 and PRDM16 in acute myeloid leukemia. Haematologica 100: e116-e117, 2015.

67. Takahashi S: Epigenetic aberrations in myeloid malignancies (Review). Int J Mol Med 32: 532-538, 2013.

68. Jo A, Mitani S, Shiba N, Hayashi Y, Hara Y, Takahashi H, Tsukimoto I, Tawa A, Horibe K, Tomizawa D, et al: High expression of EVI-1 and MEL1 is a compelling poor prognostic marker of pediatric AML. Leukemia 29: 1076-1083, 2015.

69. Lavallée VP, Gendron P, Lemieux S, D'Angelo G, Hébert J and Sauvageau G: EVI-1-rearranged acute myeloid leukemias are characterized by distinct molecular alterations. Blood 125: 140-143, 2015. 